\title{
The Social Cost of Major Depression. A Systematic Review
}

\author{
Silvia Coretti ${ }^{1,2}$, Filippo Rumi ${ }^{2}$, Americo Cicchetti ${ }^{1,2}$ \\ ${ }^{1}$ Facoltà di Economia, Università Cattolica del Sacro Cuore, Rome, Italy \\ ${ }^{2}$ Graduate School of Health Economics and Management - Università Cattolica del Sacro Cuore, Rome, Italy \\ Correspondence: Silvia Coretti, Facoltà di Economia, Graduate School of Health Economics and Management - \\ Università Cattolica del Sacro Cuore, Rome, Italy
}

Received: November 11, 2018 Accepted: December 7, 2018 Online Published: January 25, 2019

doi:10.5539/res.v11n1p73

URL: https://doi.org/10.5539/res.v11n1p73

\begin{abstract}
Major depression (MD) is a major cause of disability and a significant public health problem due to strong physical and mental impairment, possible complications for patients (including suicides), serious social and working problems to the patient and his/her family. We provide an overview of the social cost of Major depression worldwide. We conducted a systematic literature review. Two search engines were queried. Screening of records and summary of evidence was performed by two researchers blindly. The review was conducted in accordance with the standards of the PRISMA guidelines.

Twenty studies met the inclusion criteria. Despite the heterogeneity in terms of population, setting and estimation techniques, the studies showed that the largest share of the burden of disease is represented by indirect costs. Among direct healthcare costs, inpatient care represents the most significant item, followed by outpatient care. The average total direct cost of depression ranges between $€ 508$ and $€ 24069$, depending on the jurisdiction where the analysis was run and the range of cost items included. Indirect costs range between $€ 1963$ and $€ 27364$.

Evidence on the cost of MD in some countries is currently lacking. A deeper understanding of the drivers of the economic burden of disease is a crucial starting point for studies concerned with the cost-effectiveness of new treatment strategies.
\end{abstract}

Keywords: cost of illness, economic burden, depressive disorder, mayor depression, social cost

\section{Background}

Depressive disorders are part of mood disorders and include major depressive disorder (single episode or recurrent major disorder), dysthymia and depressive disorder not otherwise specified. These disorders may result from a specific triggering event such as a sudden bereavement or a financial reverse. In case of recurrent depressive episodes, generally the first episode is due to a stressful event or situation, while in later episodes' connection with a triggering factor may be less obvious or apparently absent. In some cases, the side effects of certain drugs and other pathologies, such as cardio-vascular events or cancer, may contribute to the appearance of depressive disorders. The depressive episode is characterized by symptoms such as mood swings, loss of interest in most activities of daily life, alterations in weight, appetite and sleep, agitation, decreased self-esteem, disturbances concentration, thoughts of death or suicidal behavior; to be that the psychiatric disorder has to last at least two weeks (First, 1994).

Major depressive disorder (MDD) is one of the diseases with the highest impact in Europe and worldwide, with at least 350 million people currently living with depression. According to the World Health Organization (WHO), depression is a major cause of disability and a significant public health problem resulting in strong physical and mental impairment, possible complications for patients (including suicides), serious social and working problems to the patient and his/her family, increased utilization of health care resources and direct and indirect personal and societal costs (Word Health Organization, 2012).

Evidence upon the epidemiological pattern of MDD worldwide is still ambiguous. In 2004, a large epidemiological study was run in 6 European countries, namely Belgium, France, Italy, Germany, the Netherlands, and Spain. Overall, 21425 individuals, aged 18 and older were interviewed between 2001 and 2003. Fourteen per cent of interviewees reported a lifetime history of any mood disorder, $13.6 \%$ any anxiety disorder and $5.2 \%$ a lifetime history of any alcohol disorder. Among mood disorders, major depression and specific phobia were the most common psychiatric disorders with almost $13 \%$ of patients reporting lifetime history of MDD and 4\% experiencing it in the past 12 months (Alonso et al., 2004). More recently, a systematic review investigated the epidemiology of MDD worldwide estimating a point prevalence of 
4.7\% (4.4-5.0\%), adjusted for methodological differences among the included studies and a pooled annual incidence of 3.0\% (2.4-3.8\%). Prevalence of MDD ranges between 8.6\% (5.2-14.0) observed in South Asia and 3.7\% (3.1-4.3) observed in North America. Estimates for Western Europe are slightly lower than those reported for Eastern and Central Europe (4.7 vs 5.1) (Ferrari et al., 2013).

Depressive disorders, unlike many other diseases, affect patients even at an early age and women more commonly than men, despite it is estimated that a 1.5 out of ten women become depressed postpartum, and this compromises the ability to curate the baby, by producing long term issues related to the growth and development of the child. In severe cases, depression may lead to suicide, one million people per year commit suicide,,of depression and for every death are estimated about 20 failed attempts (Word Health Organization, 2012; Hardt et al., 2015).

In several studies, MDD was proved to severely impair individuals' functioning in a number of ways, by negatively affecting not only mental well-being but also perceived physical functioning and bodily pain, as well as general health perception. This, in turn, threatens individuals' family and social life, often resulting in insulation (Saarijärvi, Salminen, Toikka, \& Raitasalo, 2002; Riihimäki et al., 2016; Papakostas et al., 2004). For this reason, MDD induces a broad decline in patients' quality of life, which is not easily captured by traditional clinical scales but should be always taken into account through generic instrument (Rapaport, Clary, Fayyad\&Endicott, 2005).

Patients with depression cannot play a "normal role" in their lives and they often have to face a hostile social environment. Typically, patients feel different and not accepted. MDD is most often associated with low self-esteem and feelings of worthlessness that induces self-discrimination (early-discrimination) (Papakostas et al., 2004).

In the US, MDD is the main cause of disability for individuals aged 15 and 44 years, by generating about 400 days lost for disability (DALYs), more than any other physical or mental disorder. Such a huge impact on patients' ability to function, was found to generate a huge economic burden in terms of direct and indirect costs, which was investigated in two studies focusing on the pattern of costs associated with depressive disorders between 1990 and 2000 and between 2005 and 2010, respectively (Greenberg et al., 2003; Greenberg, Fournier, Sisitsky, Pike \& Kessler, 2015). In the former study, authors found that the overall weight of major depression, bipolar disorder and dysthymia in 2000 amounted to $\$ 83.1$ billion, of which $\$ 26.1$ billion for direct medical costs, $\$ 5.4$ billion attributable to mortality from suicide and $\$ 51.5$ billion of losses productivity-related absence from work or reduced work productivity, caused by the diseases (Greenberg et al., 2003; Greenberg et al., 2015). This piece of evidence was then updated by the latter study, in which authors found that the overall economic burden of MDD had been rising from 2000 to 2010 by $21.5 \%$ and that the management of comorbidities was playing a prominent role in this growth (Greenberg et al., 2015).

Despite this remarkable social and economic burden, MDD is still a strongly underdiagnosed and under treated condition. According to the WHO, only one on 10 patients with depressive disorder, receives adequate treatment and support despite the existence of many treatments to improve the patient's prognosis (Word Health Organization, 2012). For instance, a large survey performed in Italy including 5.566 individuals, found that $62,7 \%$ of patients with MDD and 83,9\% of those with minor depressive symptoms had never asked for a specialist advice upon their own condition. Moreover, $34,2 \%$ of patients with MDD receiving medical advice had not received any prescription, while 36,8\% had started medical treatments more than once. Furthermore, the number of consultations, of pharmacological treatments and diagnostics were three times bigger in patients with MDD compared to individuals without depressive symptoms, while the number of absences from work lasting more than one week occurring in the last 6 months was four times bigger (Battaglia, Dubini, Mannheimer \& Pancheri, 2004). In existing literature, this important diagnostic and therapeutic unmet need has been attributed not only to the poor awareness, social stigma and the mistrust in pharmacological treatments, but also to the great variability in doctors' ability to correctly recognize and interpret the disease symptoms (Lasalvia et al., 2013).

On the other hand, a number of innovative treatment strategies for the management of MDD is becoming available (Thase, 2017). However, in many national settings, especially those characterized by the presence of a publicly funded health care systems, the market access of new drugs is usually conditional on the assessment of its economic impact (Paris \&Belloni, 2013). As a matter of fact, in the presence of limited health care resources and potentially infinitive health care needs, each decision made concerning the reimbursement of a new drug in a clinical area has a high opportunity cost, in terms of treatments forgone for other diseases. For these reason, economic evaluations of health care technologies are increasingly used also by regulatory agencies to inform decision making providing an assessment of the cost-benefit balance. However, an exhaustive assessment of a health technology (i.e. drug, procedure or medical device) cannot be performed without a deep understanding of the composition of the costs of the condition.

Cost-of-illness is the most ancient economic evaluation technique used in the health sector. Its aim is to measure the economic burden of illness to society. It is a descriptive study that can provide information to support the political process providing an estimate of the true cost of the condition to society, an analysis of the main cost components and their incidence over total costs as well as indication of the different subjects who actually bear the costs (Tarricone, 2006). 
The aim of this systematic review is to provide an overview of the social cost of MDD worldwide, focusing on the composition of the overall economic burden. Looking at the cost composition, we provide insights on the current patterns and challenges in the management of these patients.

\section{Materials and Methods}

\subsection{Data Sources \& Research Strategy}

In order to achieve the objectives of the study a search string was built and run on two engines, namely PubMed and EBSCOhost, including the following databases: Medline, CINAHL, Cochrane Database, Health Technology Assessments and NHS Economic Evaluation Database. The search string combined the following key words: "depressive disorder", "major depr*", depression, "economic burden", burden, cost, "cost of illness", "disease cost". Appropriate Boolean operators were utilized to combine key words together. In order to not miss any potentially relevant record, some Mesh terms were identified and looked up on Pubmed: "Depressive Disorder", "Depression", "Depressive Disorder, Major", "Cost of Illness", "Costs and Cost Analysis", "Cost Control", "Health Care Costs".

The literature search was limited to papers with full text available, published in English language during the last ten years. The study population was limited to adult humans and key words needed to be mentioned in the title and/or in the abstract.

\subsection{Study Selection Process and Inclusion/Exclusion Criteria}

The eligibility of the studies was ascertained by two steps: after excluding duplicates, a preliminary screening was done on the basis of title and abstract. Afterward, the studies included in this prior assessment were selected based on the full text at the second screening. Each record retrieved was assessed by two junior researchers blindly. A third reviewer intervened in case of doubt or disagreement.

Eligibility was assessed against the following criteria: $i$ ) Study type: primary report of original research; reviews, systematic reviews, editorials and protocol papers were excluded. ii) Type of analysis: cost of illness, economic studies, clinical studies analyzing costs based on either retrospective administrative data or surveys were deemed eligible. iii) Data reported: eligible papers had to include enough detail on cost data and their sources; $i v$ ) Full text availability: the full text of eligible papers needed to be available to reviewers; corresponding authors were contacted where necessary. The Consolidated Health Economic Evaluation Reporting Standards (CHEERS) 24-item checklist was used to evaluate the quality of the studies reviewed (Husereau et al., 2013).

\subsection{Data Extraction, Analysis and Reporting}

Standardized data extraction forms were used. Each record yielded by the search strategy was attached a progressive ID number, in order to be easily tracked. For each paper, regardless of its subsequent inclusion in the review, first author, title, source and study design were recorded.

For included studies, information on population, study design, cost data sources estimates of direct and indirect costs were collected. After careful reading, cost items were classified into more general cost categories in order to allow for comparisons across studies. Costs of different currencies have been converted to Euros 2015 and adjusted by the consumer price index. Where possible, values have been presented per patient per year.

The reporting was compliant with the standards of the "Preferred Reporting Items for Systematic Reviews and Met-Analyses" (PRISMA) guidelines (Liberati et al., 2015).

\section{Results}

\subsection{Search Results}

Overall, the search strategy yielded 12910 records. After the removal of 4271 duplicates, another 3412 papers were considered not relevant based on the reading of the title. The remaining 956 abstracts were assessed for eligibility. Of these, 793 were excluded for not complying with eligibility criteria and 163 full texts were reviewed. The reading of full texts led to the exclusion of 143 articles have been excluded for the following reasons: 84 reported cost-effectiveness analyses and not original cost analysis assessments, 39 referred to populations not relevant to the aim of the current review, and 21 focused on clinical and/or epidemiological aspects of MDD. Twenty records eventually met our inclusion criteria (Greenberg et al., 2015; Birnbaum et al., 2010; Stensland, Jacobson \& Nyhuis, 2007; Ivanova et al., 2011; Unützer et al., 2009; Olchanski et al., 2013; François, Despiégel, Maman, Saragoussi \&Auquier, 2010; Vasiliadis et al., 2013; Lepine, Moreno, Campos \& Couttolenc, 2012; Tomonaga et al., 2013; Sobocki, LeKander, Borgström, Ström \& Runeson, 2007; von Knorring, Akerblad, Bengtsson, Carlsson, \& Ekselius, 2006; Ekman, Granström, Omérov, Jacob \& Landén, 2013; Salvador-Carulla et al., 2011; Kleine-Budde et al., 2013; Bosmans et al., 2010; Sado et al., 2011; Hu, He, Zhang \& Chen, 2007; Ho, Mak, Chua, Ho, Mak, 2013; Chang, Hong \& Cho, 2012).

The study selection process is depicted in Figure 1. 


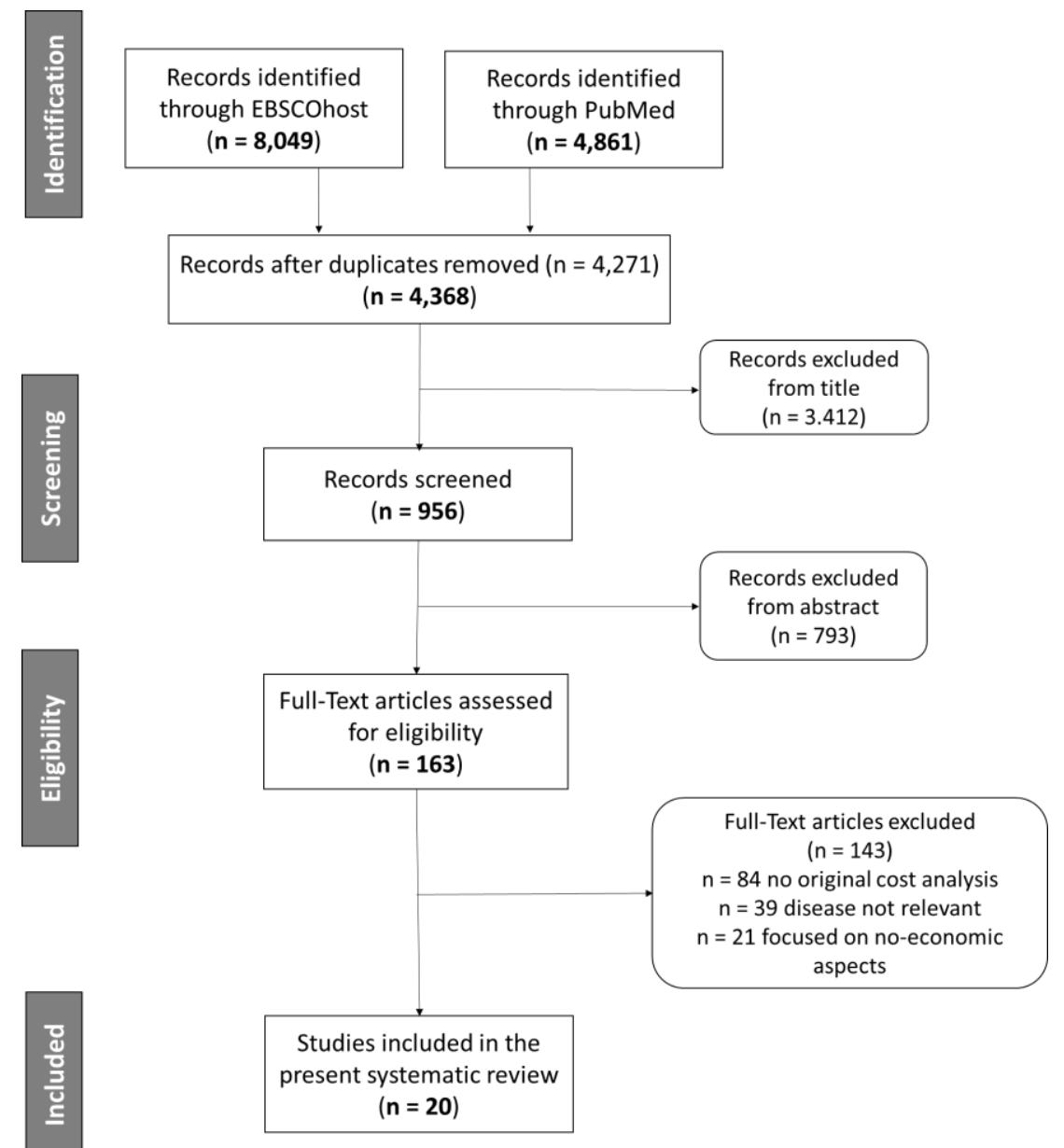

Figure 1. Flow chart of systematic literature review

Nine of the studies included in the current review were conducted in America, 7 in Europe and 4 in Asia. General features of the studies are listed in Table 1 reporting the study year (i.e. the year that data refer to), the size and age of the study population, the case definition, the study perspective and the cost data sources.

Fourteen out of 20 studies observed patients for a time span longer than one year (Greenberg et al., 2015; Birnbaum et al., 2010; Ivanova et al., 2011; Unützer et al., 2009; Olchanski et al., 2013; François et al.,2010; Vasiliadis et al., 2013; Lepine et al., 2013; Tomonaga et al., 2013; von Knorring et al., 2006; Ekman et al., 2013; Kleine-Budde et al., 2013; Bosmans et al., 2010, Ho et al., 2013). Only in 3 out of 20 studies (Birnbaum et al., 2010; Vasiliadis et al., 2013; Hu et al., 2007), eligible patients were selected though physician diagnosis, whereas in the remaining cases diagnosis-related classifications were used.

Eleven studies (Birnbaum et al., 2010; Greenberg et al., 2015; Tomonaga et al., 2013; Sobocki et al., 2005; von Knorring et al., 2006; Ekman et al., 2013; Salvador-Carulla, 2011; Sado et al., 2011; Hu et al., 2007; Ho e5t al., 2013; Chang et al., 2012) adopted either a patient or societal perspective, including estimation of both health care direct costs and productivity losses. The remaining papers investigated the cost of MDD under the third party payer perspective. Of these, 3 (Unützer et al, 2009; Vasiliadis et al., 2013; lepine et al., 2012) adopted the public payer point of view and 6 adopted the insurer perspective (Stensland et al., 2004; ivanova et al., 2011; Olchanski et al., 2013; François et al., 2010; Kleine-Budde et al., 2013; Bosmans et al., 2010).

All the studies included assessed direct costs (i.e. costs directly related to the MD treatment) exhaustively, by organizing them in the following labels: inpatient costs, outpatient costs, depressive drugs, non-depressive drugs and ancillary care. Inpatient costs have been aggregated from the following cost items: hospital, hospital stays, hospitalizations whereas outpatient costs involve ambulatory, day hospital, primary care, specialized care, general practice and psychiatric consultations. Finally, ancillary care includes diagnostic (e.g. laboratory tests, radiology, genetic testing, diagnostic imaging), therapeutic (rehabilitation to physical and occupational therapy, massage, chiropractic services, speech therapy), and custodial services (hospice care, long-term acute care, nursing facilities and urgent care).

Indirect costs (i.e. costs to the society generated by the inability to work due to the disease) were evaluated in terms of 
suicides and loss of work productivity generated by either presentism or absenteeism. The loss of productivity was always calculated through the human capital approach. Despite the heterogeneity in terms of population and geographical location and the difference in costs estimation techniques, all the included studies show an important weight of indirect costs within the overall economic burden. Detail on cost data is reported in Table 2 including values per patient per year.

Table 1. Main characteristics of included and relevant studies

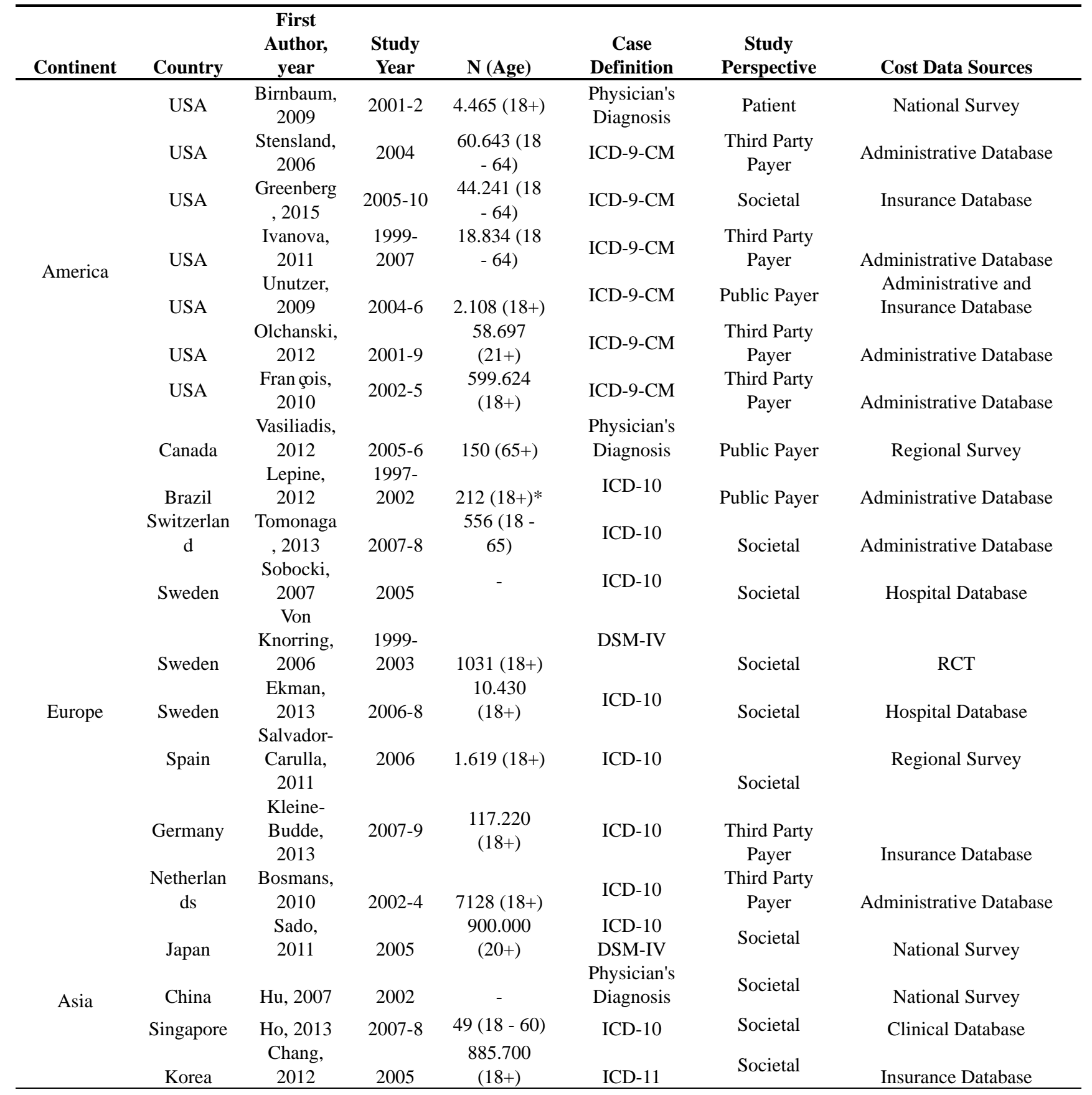


Table 2. MDD direct and indirect costs per patient/year

\begin{tabular}{|c|c|c|c|c|c|c|c|c|c|c|c|}
\hline \multirow{2}{*}{$\begin{array}{l}\text { First author, } \\
\text { Year, Country }\end{array}$} & \multicolumn{6}{|c|}{ Direct health care costs $(€)$} & \multicolumn{3}{|c|}{ Indirect costs $(€)$} & \multirow[b]{2}{*}{ Total } & \multirow[b]{2}{*}{ Suicide } \\
\hline & 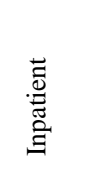 & 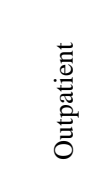 & 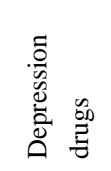 & 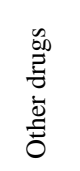 & 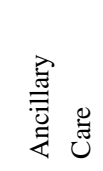 & 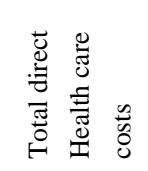 & 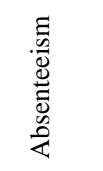 & 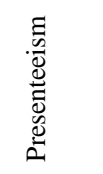 & 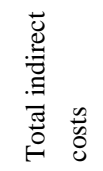 & & \\
\hline Birnabaum,2009, US & 1183 & 215 & 186 & N/A & 347 & 1931 & 2211 & N/A & 2211 & 4142 & N/A \\
\hline Stensland,2006, US & 2619 & 1158 & 439 & 82 & N/A & 4298 & N/A & N/A & N/A & N/A & N/A \\
\hline Greenberg,2015, US & 2509 & 1226 & 1019 & 1540 & 483 & 6777 & 11977 & 1963 & 13941 & 20718 & 478390 \\
\hline Ivanova, 2011, US & 4364 & 8260 & 2720 & 2614 & N/A & 17960 & N/A & N/A & N/A & N/A & N/A \\
\hline Unutzer, 2009, US & 9454 & 8470 & N/A & N/A & 6145 & 24069 & N/A & N/A & N/A & N/A & N/A \\
\hline Olchanski, 2012, US & 902 & 5415 & 931 & 1409 & N/A & 8656 & N/A & N/A & N/A & N/A & N/A \\
\hline François, 2010, US & 2650 & 2682 & 903 & N/A & 284 & 6518 & N/A & N/A & N/A & N/A & N/A \\
\hline $\begin{array}{l}\text { Vasiliadis, 2012, } \\
\text { Canada }\end{array}$ & 618 & 1459 & 1224 & N/A & N/A & 3302 & N/A & N/A & N/A & N/A & N/A \\
\hline Lepine, 2012, Brazil & 323 & 137 & 1171 & N/A & 778 & 2409 & N/A & N/A & N/A & N/A & N/A \\
\hline $\begin{array}{l}\text { Tomonaga, 2013, } \\
\text { Switzerland }\end{array}$ & 8701 & 1903 & 877 & N/A & $\mathrm{N} / \mathrm{A}$ & 11482 & N/A & 14359 & 14359 & 25841 & N/A \\
\hline $\begin{array}{l}\text { Sobocki, 2007, } \\
\text { Sweden }\end{array}$ & 342 & 401 & 1757 & N/A & N/A & 2501 & N/A & 5136 & 5136 & 7637 & 439705 \\
\hline $\begin{array}{l}\text { von Knorring, 2006, } \\
\text { Sweden }\end{array}$ & 641 & 1471 & 308 & 711 & N/A & 3131 & N/A & 20585 & 20585 & 23717 & N/A \\
\hline $\begin{array}{l}\text { Ekman, 2013, } \\
\text { Sweden }\end{array}$ & 792 & 1115 & 286 & N/A & N/A & 2193 & N/A & 16330 & 16331 & 18524 & N/A \\
\hline $\begin{array}{l}\text { Salvador-Carulla, } \\
\text { 2011, Spain }\end{array}$ & 3704 & 453 & 21 & N/A & N/A & 4178 & 6046 & 21318 & 27364 & 31542 & 401635 \\
\hline $\begin{array}{l}\text { Kleine-Budde, 2013, } \\
\text { Germany }\end{array}$ & 224 & 129 & 155 & N/A & N/A & 508 & N/A & N/A & N/A & N/A & N/A \\
\hline $\begin{array}{l}\text { Bosmans, 2010, } \\
\text { Netherlands }\end{array}$ & N/A & 2.312 & 571 & 599 & N/A & 3482 & N/A & N/A & N/A & N/A & N/A \\
\hline Sado, 2011, Japan & 470 & 658 & 8564 & N/A & N/A & 1692 & 8647 & N/A & 8647 & 10339 & 439705 \\
\hline $\mathrm{Hu}, 2007$, China & 113 & 221 & 75 & N/A & 179 & 588 & 138 & 133 & 272 & 860 & 2.650 \\
\hline Ho, 2013, Singapore & 575 & 202 & 480 & N/A & 192 & 1449 & 3058 & 3121 & 6178 & 7628 & N/A \\
\hline Chang, 2012, Korea & 84 & 349 & 35 & N/A & N/A & 468 & 6046 & 21318 & 27364 & 27833 & 401635 \\
\hline
\end{tabular}

\subsection{Direct Health Care Costs}

Nine out of 20 studies were performed in America (Greenberg et al., 2015; Birnbaum et al., 2010; Stensland et al., 2004; Ivanova et al., 2011; Unützer et al., 2009; Olchanski et al., 2013; François et al., 2010; Vasiliadis et al., 2013; Lepine et al., 2012). Of these, 7 (Greenberg et al., 2015; Birnbaum et al., 2010; Stenslad et al., 2004; Ivaniva et al., 2011; Unützer et al., 2009; Olchanski et al., 2013; François et al., 2010) were conducted in the US, 1 in Canada (Vasiliadis et al., 2013) and one in Brazil (Lepine et al., 2012). In these studies, among direct health care costs, inpatient care and outpatient care represent the most significant items, equaling on average $€ 2751$ ( $€ 460-€ 9454, \mathrm{SD}=€ 2813)$ and $€ 3406$ ( $€ 215-€$ $8470, \mathrm{SD}=€ 3167)$, respectively. The weight of inpatient care is heavier than that of outpatient care in five studies (Greenberg et al., 2015; Birnbaum et al., 2010; Stensland et al., 2004; Unützer et al., 2009; Lepine et al., 2012).

Studies performed in Europe showed a similar pattern of direct health care costs, and even if the amounts are different the relative impact of cost items is comparable to the one observed for the American studies. Four out of 7 articles reported a value of inpatient costs higher than outpatient costs (Tomonaga et al., 2013; Salvador-Carulla et al., 2011; Klein-Budde et al., 2013; Bosmans et al., 2010), while in the remaining 3 studies the trend is opposite (Sobocki et al., 
2007; von Knorring et al., 2006; Ekman et al., 2013). The inpatient costs have on average a value of $€ 2089$ (range: $€$ $207-€ 8701$ ) with a standard deviation of $€ 3573$. The outpatient costs have on average a value of $€ 1438$ with a standard deviation of $€ 838$, range between $€ 128$ and $€ 2312$.

In absolute terms, studies conducted in Asia show a lower burden of disease in terms of direct health care costs compared with Occidental countries, probably due to differences in the patient management strategies. The inpatient costs average $€ 376$ with a standard deviation of $€ 258$ (range: $€$ 84- $€ 575$ ), while the outpatient costs average $€ 373$ with a standard deviation of $€ 248$ (range between $€ 202$ and $€$ 658) (Sado et al., 2011; Hu et al., 2007; Ho et al., 2013; Chang et al., 2012).

Pharmaceuticals represent worldwide a share of $24 \%$ on the total direct costs of illness. Among studies performed in Northern America, the incidence of the cost of drugs on the overall cost of illness is very heterogeneous. Three studies (Greenberg et al., 2015; Ivanova et al., 2011; Vasiliadis et al., 2013) report a cost of anti-depressive and non-anti-depressive drugs which accounts for more than $30 \%$ of the overall cost per patient. Two of these studies refer to the US setting (Greenberg et al., 2015; Ivanova et al., 2011) the third one was performed in Canada (Vasiliadis et al., 2013). In another 3 studies the cost of drugs accounts more 15\% (Birnbaum et al., 2010; Stensland et al., 2004; François et al., 2010).

In Europe, on average, the impact of drugs on the overall cost of disease is $20,32 \%$, with only two studies reporting a figure higher than 30\% (von Knorring et al., 2006; Klein-Budde et al., 2013). In the studies focusing on the European setting such value is below 16\% (Tomonaga et al., 2013; Ekman et al., 2013 Bosmans et al., 2010).

Studies performed in Asia report a relatively high incidence of pharmaceuticals on the total direct cost. Pharmaceuticals account for more than $30 \%$ in Sado et al. (2011), where the cost of drugs is $€ 564$ on a total direct costs of $€ 1692$, as well as in Ho et al. (2013) where the cost of medicines is $€ 480$ on a total direct costs of $€ 1449$. In Chang et al. (2012) the incidence of pharmaceuticals on the overall cost is near to $10 \%$.

The supportive care is a costs item extremely complex and articulated, including different types of care aimed at improving the patients' quality of life, preventing or treating as early as possible the symptoms of disease, managing side effects caused by the treatment, and providing psychological, social, and spiritual support. Worldwide the supportive care costs represent, on average, the $14.25 \%$ of the total direct costs, ranging between $4 \%$ and $26 \%$. However, only six studies took this cost item into account (Greenberg et al., 2015; Birnbaum et al., 2010; Unützer et al., 2009; François et al., 2010; Bosmans et al., 2010; Ho et al., 2013).

Nonetheless, such a cost item deserves careful attention since supportive care plays a central role in the management of a disease like depression, for whom the support service activities outside of health care structures and the presence and support of a caregiver is essential for patient, both from the physical and the psychological point of view.

The average total direct cost of depression, in America ranges between $€ 1931$ and $€ 24069$, in Europe between $€ 508$ and $€ 11482$ and in Asia between $€ 380$ and $€ 1$ 692; the variation in range depending on the jurisdiction where the analysis was run and the range of cost items included by the authors.

\subsection{Indirect Costs}

The indirect costs were gathered from every article and were organized and tabulated in three cost labels: suicides, directly linked with depressive illness, and loss of work productivity, in terms of presentism (i.e. productivity loss on the work place due to poor health conditions) and in terms of absenteeism (i.e. productivity losses due to absence from work due to depression).

Only two studies performed in America have dealt with indirect costs (Greenberg et al., 2015; Birnbaum et al., 2010). Birnbaum et al. (2010) investigated, in a sample of 4465 US workforce, the presentism costs by assessing a loss of productivity per person of $€ 2211$. There was a significant correlation between MD severity and treatment usage and costs, as well as between treatment adequacy and severity. Disability increased with MD severity, while work performance decreased with MD severity. Greenberg et al. (2015) evaluated the indirect costs in term of absenteeism, by identifying a significant weight of loss of productivity on the economic burden of MD. The injury, illness and disability generated an absenteeism cost of $€ 1963$ per person, which have contributed to the increase economic impact of MD in USA (Greenberg et al., 2015).

Among the studies performed in Europe, 5 investigated also the indirect costs; the absenteeism costs averaged $€ 14330$ with a standard deviation of $€ 6104$ (range: between $€$ 6046- $€ 20$ 585), (Tomonaga et al., 2013; Sobocki et al., 2007; von Knorring et al., 2006; Ekman et al., 2013; ; Salvador-Carulla et al., 2011; Klein-Budde et al., 2013). Tomonaga et al. (2013) assessed the economic burden of depression in Switzerland, the loss of productivity was significantly associated with severity of depression, increased unemployment, disability and reduced work performance, in terms of workdays lost. The enormous impact of depression on patients' ability to work was shown, by the authors, with unemployment and 
employment rates of $25.8 \%$ and $59.2 \%$, respectively, with almost 50 workdays lost per year and an absenteeism value near to the European average. Von Knorring et al.(2006) emphasized the importance of the attention on the indirect costs and highlighted potential cost savings from a reduction in absences from work for patients responding to medical treatment, substantially lowering the indirect costs associated with the disease. Such a result is particularly relevant to the context where the study was performed, since costs for sickness absence in Sweden are high and increasing, and psychiatric disorders are the second most common reason for sick leave. In this setting, the absenteeism cost is more than $€ 20000$ per person. Another Swedish study of 2013 assessed the impact of indirect costs on the overall economic burden of MD (Ekman et al., 2013); the authors computed an absenteeism value of $€ 16331$. The indirect cost due to sick leave and early retirement was by far the largest cost item for patients with depression; treatment improvements that can bring back more patients to work, therefore, have the potential to lead to substantial cost offsets from the societal perspective. In Spain, Salvador-Carulla et al. (2011) calculated the absenteeism value with a particular attention on the difference between temporary and permanent disability. The value taken into account through this review is $€ 6046$ of absenteeism for temporary disability, instead the loss of productivity due to permanent disability has a higher cost which amounted to $€ 21$ 318.

Concerning studies performed in the Asian setting, Sado et al. (2011) assessed the presentism cost around $€ 8647$ per person. In Japan, the productivity loss of patients with depression who continue to work is enormous. In this situation, early interventions, such as effective screening along with access to appropriate treatment, could contain the social implications of this lack of productivity and work activity. The study conducted by Ho et al. (2013), in Singapore, evaluated the loss of productivity of a medium worker such as $€ 3058$ per absenteeism and $€ 3121$ per presentism. In this study, the weight of indirect cost is very relevant, representing approximately $80 \%$ of total costs per MD patient. Chang et al. (2012) estimated a social cost for morbidity of $€ 2859$ for absenteeism and $€ 4498$ for presentism for South Korea.

Among indirect cost, suicides, directly related to depressive illness, represent a tremendous burden for society. In the US, Greenberg et al. (2015) assessed the value of suicides in $€ 478390$ per patient, while in Spain and in Sweden the suicide cost was estimated in $€ 401635$ an $€ 454$ 334, respectively. In Japan, the cost per depression-related death was estimated in $€ 439705$; however, the suicide rate here is two and four times higher than in the US and in the UK, respectively (Sado et al., 2011). Considering that approximately half of the total costs of depression in Japan can be attributed to mortality costs, effective interventions to prevent suicides should be implemented. Regardless of the geographical location and the reference healthcare setting of the study, the economic burden of the suicides related to depression, exceeds $€ 400000$ per person, except for South Korea where a cost of about $€ 190$ 000/ suicide was computed (Chang et al., 2012).

Most of the studies included in this review concluded that the indirect cost is the most relevant share of the overall economic burden of major depression. Access to health care services, as well as, adequacy of the treatment received have been found to be strongly related to lower disability rates and avoidance of losses of productivity, measured in terms of absenteeism and presentism. Among indirect costs, the suicides have a significant and tremendous social impact and economic burden on the welfare system. Therefore, the investments in health and health plans related to depression may have a significant impact on social and economic system.

Figures 2 and 3 show the composition of the overall cost per patient with MD. In particular, Figure 2 only includes studies run under the third party payer perspective and, for this reason only focuses on direct health care costs. Figure 3 includes studies with a societal perspective, which focus also on indirect costs. The white dot on the secondary y axes represents the incidence of the total indirect cost (i.e. preseenteism + absenteeism) on the overall cost per patient. 


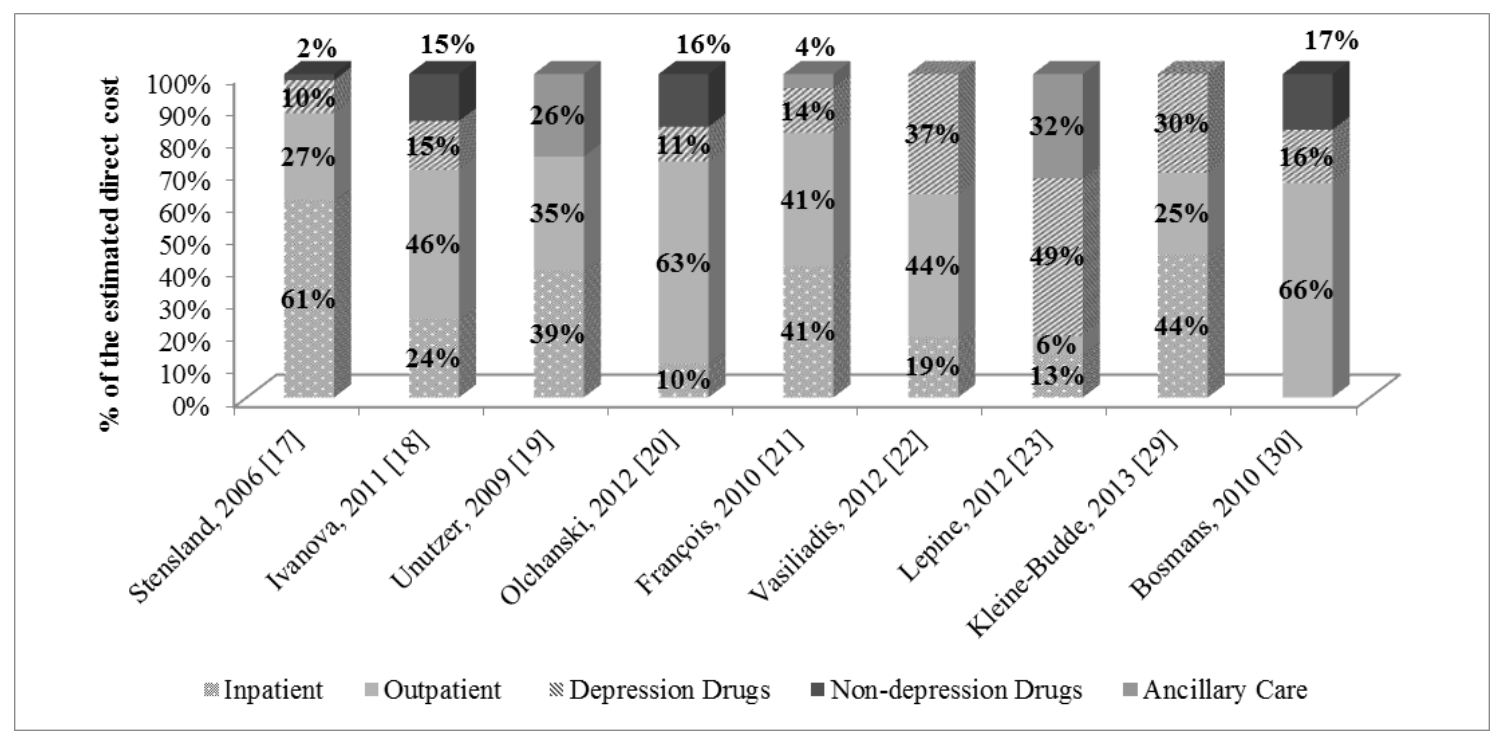

Figure 2. Direct health care cost in studies adopting the third party payer perspective

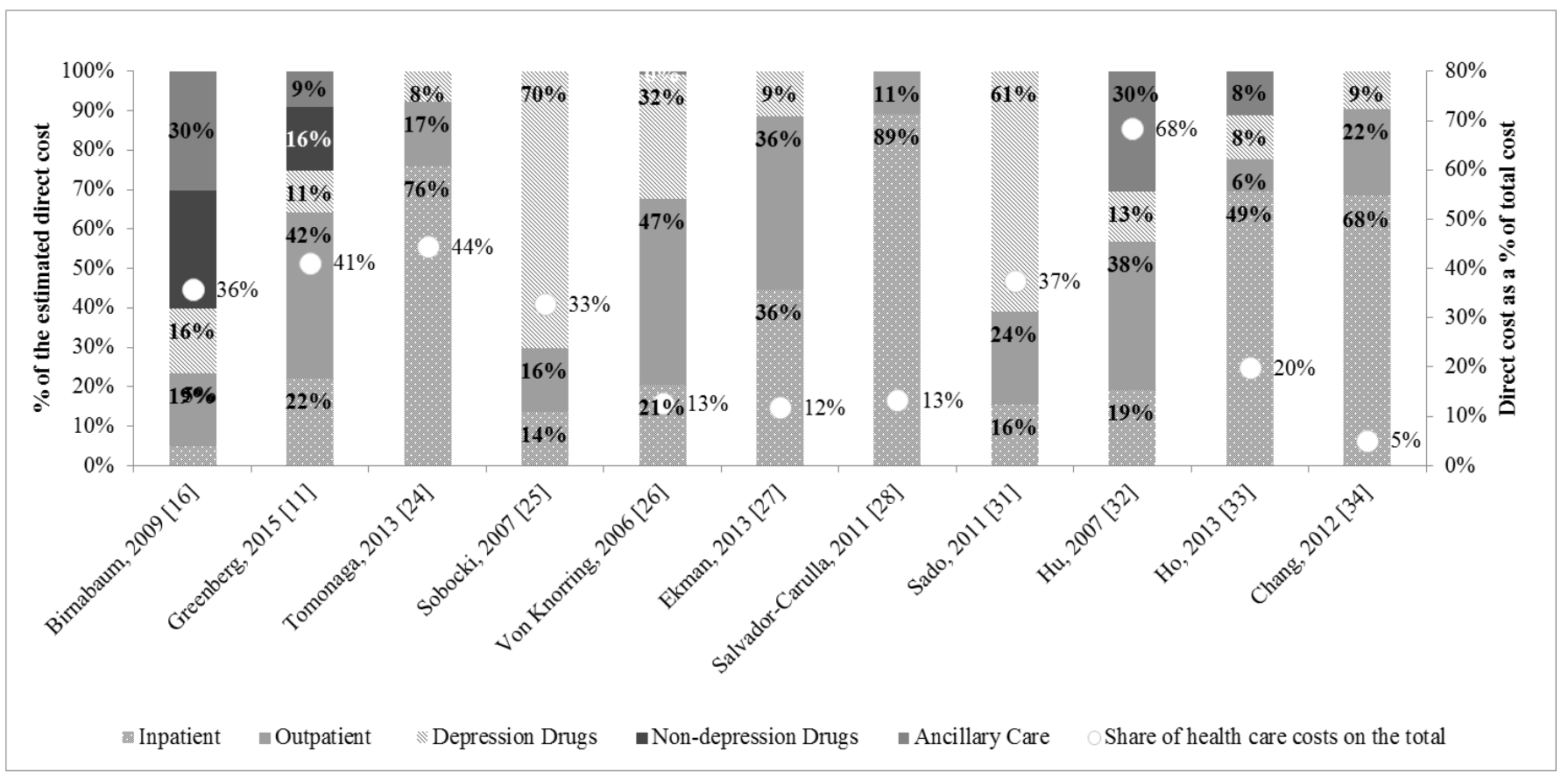

Figure 3. Direct health care cost in studies adopting the societal perspective

\section{Discussion}

Depression is a health problem of paramount importance under the clinical, societal and economic point of view. Despite its severity, the evidence shows that depression is a severely under diagnosed disease in many countries and, very often, even when diagnosed, it is not properly treated.

The current review shed light on the societal cost of major depression worldwide. When indirect costs are taken into account, they represent the most significant share of the total cost of depression. These costs include loss of productivity for patients resulting from working days losses or decreased productivity in working places, losses of productivity for care givers and future income forgone due to suicides. Where estimated, the cost of loss of productivity at work, weighs more than the loss of productivity for absence from work. Despite this huge burden, indirect costs are usually overlooked in economic analysis on treatment strategies for major depression and even when computed they are hardly comparable across settings, due to the deep differences across welfare systems. 
A recent study estimated the social cost of cognitive impairment in working patients with MD. Using a model the authors estimated the annual productivity losses due to MD-related cognitive impairment, by relating data concerning absenteeism and presentism to patients' cognitive function measured by a 10-point scale then converted into a Sheehan Disability Scale (SDS). Authors found a loss of $€ 1129$ per patient per month attributable only to cognitive impairment. In the sensitivity analysis such a figure ranged between $€ 1104$ and 1464 according to the scale utilized to measure cognitive impairment. Comparted to an healthy individual, a patient with major experiences a loss of productivity of $42,27 \%$ (Coretti et al., 2016).

Among direct health care costs, the most important items are hospitalizations and visits, whereas drugs represent a modest share of total direct cost. To this end, existing literature as well as real world evidence show that in the area of depression and mental disorders in general, a big problem of under-treatment or mistreatment exists. Some authors attribute this phenomenon to the lack of awareness of clinicians in the diagnostic phase and in the definition of the most appropriate pathway for the patient. Another branch of literature attributes such phenomenon to the lack of patient's compliance due to the reluctance to undertake the therapy to avoid social stigma (Lasalvia et al., 2013; Bataglia et al. 2004). Nonetheless, research that aims to design and evaluate comprehensive interventions aimed at minimizing stigma and social exclusion and facilitating access to care is still lacking (Lasalvia et al., 2013; Evans-lacko, Brohan, Mojtabai \& Thornicroft, 2012).

Our study carries a few limitations that deserve mention. Firstly, the choice of strict inclusion criteria has limited the scope of the analysis to studies published in English in the last ten years. Such a choice may have determined the omission of potentially relevant pieces of evidence. However, usually studies published in international journals in English language have higher quality. Moreover, the choice of restricting the time span to the last ten years, was done because considering long time spans carries the risk of including cost-of-illness studies very heterogeneous in terms of state of art of clinical and technological practice. Another limitation is that the summary of evidence is only narrative. The great heterogeneity of the included studies prevented us from performing a quantitative synthesis of results.

On the other hand, our study sheds new light on the burden of this condition and the challenges for the management of patients with MD. In the current review, we found that spending for hospital admission is higher in contexts where spending on medical treatments is low. Appropriateness of medical treatments prescribed for MD is another topic that deserves attention in future research. A study conducted in Northern Italy showed that, regardless of the type drug utilized patients receiving medical treatments exhibited a lower hospitalization rate (Deambrosis, Terrazzini, Giusti, Pullia,\&Chinellato, 2007). Moreover, a case-control study performed in Sardinia (Italy) showed that patients diagnosed with major depression require a higher health care resource consumption compared to the non-depressed individuals and to healthy ones. In addition, an increase in direct health care cost, such as drugs and hospital admission was found in patients without adequate medical treatment (Carta, Hardoy, Kovess, Dell’Ossoand Carpiniello, 2003).

\section{Conclusions}

Despite being one of the major causes of disability in the world, major depression is still largely under-diagnosed and undertreated, also due to the social stigma associated with it. Evidence on epidemiology of the disease as well as on its economic burden is now very fragmentary and poorly generalizable, due to the deep differences across settings. Nonetheless, our analysis allowed to draw some conclusions: i) hospitalization is often the most relevant cost item when looking at direct healthcare costs; the larger this item is the smaller is the expenditure on medical treatments. This relation probably means that complications are more usual when the medical management of disease is poor or inadequate. This aspect deserves a deeper investigation in future research; ii) most of the economic burden of disease is represented by productivity losses resulting in the indirect costs; this is due to the characteristics of the disease which affects individuals in their working age; iii) productivity losses from preseenteism are usually heavier than those resulting from absenteeism. These aspects should be further investigated in future research and need to be taken into account when performing economic evaluations of treatment strategies for patients with MD.

\section{References}

Alonso, J., Angermeyer, M. C., Bernert, S., Bruffaerts, R., Brugha, T. S., \& Bryson, H. (2004). Prevalence of mental disorders in Europe: results from the European Study of the Epidemiology of Mental Disorders (ESEMeD) project. Acta Psychiatrica Scandinavica, 109(420), 21-7.

Battaglia, A., Dubini, A., Mannheimer, R., \& Pancheri, P. (2004). Depression in the Italian community: epidemiology and socio-economic implications. International clinical psychopharmacology, 19(3), 135-142. https://doi.org/10.1097/00004850-200405000-00003

Birnbaum, H. G., Kessler, R. C., Kelley, D., Ben-Hamadi, R., Joish, V. N., \& Greenberg, P. E. (2010). Employer burden of mild, moderate, and severe major depressive disorder: mental health services utilization and costs, and work performance. Depression and anxiety, 27(1), 78-89. https://doi.org/10.1002/da.20580 
Bosmans, J. E., de Bruijne, M. C., de Boer, M. R., Van Hout, H., Van Steenwijk, P., \& Van Tulder, M. W. (2010). Health care costs of depression in primary care patients in The Netherlands. Family practice, 27(5), 542-548. https://doi.org/10.1093/fampra/cmq033

Carta, M. G., Hardoy, M. C., Kovess, V., Dell'Osso, L., \& Carpiniello, B. (2003). Could health care costs for depression be decreased if the disorder were correctly diagnosed and treated?. Social psychiatry and psychiatric epidemiology, 38(9), 490-492. https://doi.org/10.1007/s00127-003-0662-z

Chang, S. M., Hong, J. P., \& Cho, M. J. (2012). Economic burden of depression in South Korea. Social psychiatry and psychiatric epidemiology, 47(5), 683-689. https://doi.org/10.1007/s00127-011-0382-8

Coretti, S., Izzo, G., Vaggi, M., Bellomo, A., Mencacci, C., \& Cicchetti, A. (2016). The Cost of Cognitive Impairment in Patient with Major Depressive Disorder. Value in Health, 19(7), A526. https://doi.org/10.1016/j.jval.2016.09.1042

Deambrosis, P., Terrazzani, G., Giusti, P., Pullia, G., \& Chinellato, A. (2007). Il costo del paziente depresso: l'esperienza della Ulss 9 di Treviso. PharmacoEconomics Italian Research Articles, 9(1), 1-8. https://doi.org/10.1007/BF03320565

Ekman, M., Granström, O., Omerov, S., Jacob, J., \& Landen, M. (2013). The societal cost of depression: evidence from 10,000 Swedish patients in psychiatric care. Journal of affective disorders, 150(3), 790-797. https://doi.org/10.1016/j.jad.2013.03.003

Evans-Lacko, S., Brohan, E., Mojtabai, R., \& Thornicroft, G. (2012). Association between public views of mental illness and self-stigma among individuals with mental illness in 14 European countries. Psychological medicine, 42(8), 1741-1752. https://doi.org/10.1017/S0033291711002558

Ferrari, A. J., Somerville, A. J., Baxter, A. J., Norman, R., Patten, S. B., Vos, T., \& Whiteford, H. A. (2013). Global variation in the prevalence and incidence of major depressive disorder: a systematic review of the epidemiological literature. Psychological medicine, 43(3), 471-481. https://doi.org/10.1017/S0033291712001511

First, M. B. (1994). Diagnostic and statistical manual of mental disorders. (4th edition). APA.

François, C., Despiégel, N., Maman, K., Saragoussi, D., \& Auquier, P. (2010). Anxiety disorders, major depressive disorder and the dynamic relationship between these conditions: treatment patterns and cost analysis. Journal of medical economics, 13(1), 99-109. https://doi.org/10.3111/13696991003591321

Greenberg, P. E., Fournier, A. A., Sisitsky, T., Pike, C. T., \& Kessler, R. C. (2015). The economic burden of adults with major depressive disorder in the United States (2005 and 2010). The Journal of clinical psychiatry, 76(2), 155-162. https://doi.org/10.4088/JCP.14m09298

Greenberg, P. E., Kessler, R. C., Birnbaum, H. G., Leong, S. A., Lowe, S. W., Berglund, P. A., \& Corey-Lisle, P. K. (2003). The economic burden of depression in the United States: how did it change between 1990 and 2000 ? Journal of clinical psychiatry, 64(12), 1465-1475. https://doi.org/10.4088/JCP.v64n1211

Hardt, J., Bernert, S., Matschinger, H., Angermeier, M. C., Vilagut, G., \& Bruffaerts, R. (2015). Suicidality and its relationship with depression, alcohol disorders and childhood experiences of violence: Results from the ESEMeD study. Journal of affective disorders, 175, 168-174. https://doi.org/10.1016/j.jad.2014.12.044

Ho, R. C., Mak, K. K., Chua, A. N., Ho, C. S., \& Mak, A. (2013). The effect of severity of depressive disorder on economic burden in a university hospital in Singapore. Expert review of pharmacoeconomics \& outcomes research, 13(4), 549-559. https://doi.org/10.1586/14737167.2013.815409

Hu, T. W., He, Y., Zhang, M., \& Chen, N. (2007). Economic costs of depression in China. Social psychiatry and psychiatric epidemiology, 42(2), 110-116. https://doi.org/10.1007/s00127-006-0151-2

Husereau, D., Drummond, M., Petrou, S., Carswell, C., Moher, D., \& Greenberg, D. (2013). Consolidated health economic evaluation reporting standards (CHEERS) — explanation and elaboration: a report of the ISPOR health economic evaluation publication guidelines good reporting practices task force. Value in Health, 16(2), 231-250. https://doi.org/10.1016/j.jval.2013.02.002

Ivanova, J. I., Birnbaum, H. G., Chen, L., Duhig, A. M., Dayoub, E. J., \& Kantor, E. S. (2011). Cost of post-traumatic stress disorder vs major depressive disorder among patients covered by medicaid or private insurance. The American journal of managed care, 17(8), 314-323.

Kleine-Budde, K., Müller, R., Kawohl, W., Bramesfeld, A., Moock, J., \& Rössler, W. (2013). The cost of depression-a cost analysis from a large database. Journal of affective disorders, 147(1-3), 137-143. https://doi.org/10.1016/j.jad.2012.10.024 
Lasalvia, A., Zoppei, S., Van Bortel, T., Bonetto, C., Cristofalo, D., \& Wahlbeck, K. (2013). Global pattern of experienced and anticipated discrimination reported by people with major depressive disorder: a cross-sectional survey. The Lancet, 381(9860), 55-62. https://doi.org/10.1016/S0140-6736(12)61379-8

Lepine, B. A., Moreno, R. A., Campos, R. N., \& Couttolenc, B. F. (2012). Treatment-resistant depression increases health costs and resource utilization. Revista Brasileira de Psiquiatria, 34(4), 379-388. https://doi.org/10.1016/j.rbp.2012.05.009

Liberati, A., Altman, D. G., Tetzlaff, J., Mulrow, C., Gøtzsche, P. C., Ioannidis, J. P., et al. (2009). The PRISMA statement for reporting systematic reviews and meta-analyses of studies that evaluate health care interventions: explanation and elaboration. PLoS medicine, 6(7). https://doi.org/10.1371/journal.pmed.1000100

Olchanski, N., Myers, M. M., Halseth, M., Cyr, P. L., Bockstedt, L., Goss, T. F., \& Howland, R. H. (2013). The economic burden of treatment-resistant depression. Clinical therapeutics, 35(4), 512-522. https://doi.org/10.1016/j.clinthera.2012.09.001

Papakostas, G. I., Petersen, T., Mahal, Y., Mischoulon, D., Nierenberg, A. A., \& Fava, M. (2004). Quality of life assessments in major depressive disorder: a review of the literature. General hospital psychiatry, 26(1), 13-17. https://doi.org/10.1016/j.genhosppsych.2003.07.004

Paris, V., \& Belloni A. (2013), Value in pharmaceutical pricing. OECD Health Working Paper No. 63. https://doi.org/10.1787/5k43jc9v6knx-en

Rapaport, M. H., Clary, C., Fayyad, R., \& Endicott, J. (2005). Quality-of-life impairment in depressive and anxiety disorders. American Journal of Psychiatry, 162(6), 1171-1178. https://doi.org/10.1176/appi.ajp.162.6.1171

Riihimäki, K., Sintonen, H., Vuorilehto, M., Jylhä, P., Saarni, S., \& Isometsä, E. (2016). Health-related quality of life of primary care patients with depressive disorders. European Psychiatry, Jul 18(37), 28-34. https://doi.org/10.1016/j.eurpsy.2016.04.008

Saarijärvi, S., Salminen, J. K., Toikka, T., \& Raitasalo, R. (2002). Health-related quality of life among patients with major depression. Nordic journal of psychiatry, 56(4), 261-264. https://doi.org/10.1080/08039480260242741

Sado, M., Yamauchi, K., Kawakami, N., Ono, Y., Furukawa, T. A., \& Tsuchiya, M. (2011). Cost of depression among adults in Japan in 2005. Psychiatry and clinical neurosciences, 65(5), 442-450. https://doi.org/10.1111/j.1440-1819.2011.02237.x

Salvador-Carulla, L., Bendeck, M., Fernández, A., Alberti, C., Sabes-Figuera, R., Molina, C., \& Knapp, M. (2011). Costs of depression in Catalonia (Spain). Journal of affective disorders, 132(1-2), 130-138. https://doi.org/10.1016/j.jad.2011.02.019

Sobocki, P., Lekander, I., Borgström, F., Ström, O., \& Runeson, B. (2007). The economic burden of depression in Sweden from 1997 to 2005. European Psychiatry, 22(3), 146-152. https://doi.org/10.1016/j.eurpsy.2006.10.006

Stensland, M. D., Jacobson, J. G., \& Nyhuis, A. (2007). Service utilization and associated direct costs for bipolar disorder in 2004: an analysis in managed care. Journal of Affective Disorders, 101(1-3), 187-193. https://doi.org/10.1016/j.jad.2006.11.019

Tarricone, R. (2006). Cost-of-illness analysis: what room in health economics?. Health policy, 77(1), 51-63. https://doi.org/10.1016/j.healthpol.2005.07.016

Thase, M. E. (September, 2017). Solving clinical challenges in major depression. Paper presented at the Psych Congress, New Orleans, LA.

Tomonaga, Y., Haettenschwiler, J., Hatzinger, M., Holsboer-Trachsler, E., Rufer, M., Hepp, U., \& Szucs, T. D. (2013). The economic burden of depression in Switzerland. Pharmacoeconomics, 31(3), 237-250. https://doi.org/10.1007/s40273-013-0026-9

Unützer, J., Schoenbaum, M., Katon, W. J., Fan, M. Y., Pincus, H. A., Hogan, D., \& Taylor, J. (2009). Healthcare costs associated with depression in medically ill fee-for-service Medicare participants. Journal of the American Geriatrics Society, 57(3), 506-510. https://doi.org/10.1111/j.1532-5415.2008.02134.x

Vasiliadis, H. M., Dionne, P. A., Préville, M., Gentil, L., Berbiche, D., \& Latimer, E. (2013). The excess healthcare costs associated with depression and anxiety in elderly living in the community. The American Journal of Geriatric Psychiatry, 21(6), 536-548. https://doi.org/10.1016/j.jagp.2012.12.016

von Knorring, L., Åkerblad, A. C., Bengtsson, F., Carlsson, Å., \& Ekselius, L. (2006). Cost of depression: effect of adherence and treatment response. European psychiatry, 21(6), 349-354. 
https://doi.org/10.1016/j.eurpsy.2006.04.005

Word Health Organization. (2012). Depression, a Hidden burden. Retrieved from: http://www.who.int/mental_health/management/depression. Last access July 2016

\section{Copyrights}

Copyright for this article is retained by the author(s), with first publication rights granted to the journal.

This is an open-access article distributed under the terms and conditions of the Creative Commons Attribution license (http://creativecommons.org/licenses/by/4.0/). 